\title{
Correction: Effect of the pigment-free optical zone diameter of decorative tinted soft contact lenses on visual function
}

Jung JW, Kim SM, Han SH, et al. Effect of the pigment-free optical zone diameter of decorative tinted soft contact lenses on visual function. Br J Ophthalmol 2016;100:633-37.

In the Funding statement, the grant number is incorrect. "Yonsei University College of Medicine for 2014 (6-2014-0036)." should read "Yonsei University College of Medicine for 2014 (6-2014-0159)."

Br J Ophthalmol 2016;100:1016. doi:10.1136/bjophthalmol-2015-306731corr1 